\title{
MELHORAMENTO DA EFICIÊNCIA E GESTÃO DAS EXPLORAÇÕES DE BOVINOS DE CARNE EM PORTUGAL
}

\author{
Maria José Palma Lampreia Dos-Santos* \\ Pedro Damião de Sousa Henriques**
}

RESUMO: O principal objetivo deste estudo é analisar os níveis de eficiência das explorações de produção de bovinos de carne em Portugal, nas regiões do Alentejo e Beira Interior e identificar a sua relação com as características das explorações agrícolas e com as suas práticas de gestão de forma a melhorar a sua eficiência. A metodologia baseia-se no Data Envelopment Analysis (DEA) para determinar a eficiência técnica das explorações e na regressão múltipla logarítmica para analisar os determinantes da eficiência. Os resultados mostram que as explorações de bovinos de carne podem aumentar a sua eficiência se adotarem melhores práticas de gestão, nomeadamente, se reduzirem a contratação de mão de obra não especializada e procederem à aquisição e manutenção adequada de capital fixo adequado à atividade. Sugere-se o reforço das medidas de Política Agrícola Comum e nacionais que promovam a renovação, a qualificação dos recursos humanos e a sua capacitação na gestão do setor, facilitando ainda e reforçando os mecanismos de entrada de novos jovens empresários agrícolas com formação acadêmica adequada. Conclui-se que o apoio da PAC e das políticas públicas de incentivos ao investimento de capital fixo inovador e de qualificação e requalificação de recursos humanos é fundamental para a melhoria das práticas de gestão e da promoção dos sistemas extensivos de produção de carne de bovino em Portugal.

PALAVRAS-CHAVE: Data Envelopment Analysis (DEA); Eficiência técnica; Gestão; Portugal.

\section{IMPROVING EFFICIENCY AND MANAGEMENT OF BEEF CATTLE FARMS IN PORTUGAL}

ABSTRACT: The main aim of this paper is to analyze the efficiency of beef cattle in Portugal, in the Alentejo and Beira Interior and identify their relationship with the

\footnotetext{
${ }^{*}$ Doutor em Economia, Docente Adjunta da Escola Superior de Comunicação Social do Departamento de Ciências Sociais - Instituto Politécnico de Lisboa, Portugal e Investigadora Integrada do ISCTE-IUL- DINÂMIA'CET, Lisboa, Portugal. E-mail: mjpl1963@gmail.com

** Doutor em Economia Agrária, Docente Associado da Universidade de Évora, Portugal, Colaborador do CEGAGE - Universidade de Évora, Portugal.
} 
characteristics of the farm and its management practices in order to improve their efficiency. The methodology is based on Data Envelopment Analysis (DEA) in order to determine the technical efficiency of farms and logarithmic multiple regression to analyze the determinants of efficiency. The results show that beef cattle farms can increase their efficiency if they adopt better management practices, namely, to reduce hiring and unskilled labor, and invest in new investments adjusted to the activity. Strengthening measures of the national and Common Agricultural Policy (CAP) is suggested to promote the qualification of human resources and their management skills and invest in education in the sector with adequate academic degrees on agricultural economics. The conclusions also suggest the reinforcement of public policies in order to increase the innovation at the sector and to promote this beef production systems environmental sustainable in Portugal.

KEY WORDS: Data Envelopment Analysis (DEA); Management; Portugal; Technical efficiency.

\section{INTRODUÇÃO}

A produção de bovinos de carne em Portugal assegura cerca de $44 \%$ da procura interna nacional. Assim, Portugal continua a importar mais de metade da carne de bovino do que consome. Na região Alentejo, ao Sul de Portugal, concentrase majoritariamente a produção de carne de bovino (cerca de 39\% da produção nacional), seguido em ordem de importância da região da Beira Interior. Apesar disso, a produção pode ser incrementada, pois o país possui condições edafo-climáticas e recursos humanos capazes de contribuir para o seu auto-aprovisionamento, nomeadamente, no Alentejo e na Beira Interior.

O Alentejo é uma região mediterrânea onde dominam as grandes planícies nas quais os sistemas extensivos de bovinos de carne podem facilmente desenvolverse, em muitos casos associados a sistemas agro-silvo-pastoris sustentáveis, onde domina o montado (Querqus suber L.) típico e único em toda a região Mediterrânica. A Beira Interior faz fronteira com o Alentejo e desta forma as suas características prolongam-se medianamente.

Relativamente aos recursos humanos, Portugal tem igualmente feito um esforço notório de classificação da sua população juvenil estando neste momento a atingir o objetivo de cumprimento da Estratégia de Lisboa e da Estratégia Europa, que 
pretende que em 2020 a população até 30 anos, formada com graus de licenciatura, atinja os 40\% (FONSECA; DIZ; DOS-SANTOS, 2016). O país possui atualmente um grande número de gestores e de engenheiros agrônomos. Apesar disso, não existe neste momento formação específica ao nível da gestão agrícola ou da economia agrícola nas instituições de nível superior portuguesas.

A produção de carne de bovino tem vindo a diminuir ligeiramente nos últimos anos, o que se deveu, principalmente, à diminuição do número de explorações (DOS-SANTOS et al., 2013), às obrigações no âmbito da Política Agrícola Comum (PAC) e reforço do regime fiscal português, associadas também a uma ligeira diminuição do subsídio aos bovinos, bem como às inerentes e crônicas dificuldades de comercialização dos produtores. A diminuição do número de explorações, prende-se por um lado, com a dificuldade de rejuvenescimento da atividade, associadas à extinção de explorações devido ao envelhecimento dos produtores e, por outro, à transformação e conversão recente de alguns terrenos em regadio na região de Alqueva acabam por ser substituídas por culturas de regadio no âmbito do novo regadio do perímetro de Alqueva com 110 mil novos hectares recentemente construído. Por outro lado, a baixa rentabilidade da produção de bovinos, com caráter cíclico devido, principalmente, à existência de um mercado de oligopsónio, regra geral, com produtores com fraco poder negocial e com cadeias de comercialização longas. Também as extensões das obrigações burocráticas decorrentes da legislação em vigor no âmbito da PAC para o setor aumentaram, ou mantêm elevada carga burocrática, se tivermos em consideração que estamos perante agricultores com pouco letramento e idade avançada. Além destas medidas a componente fiscal também se agravou no âmbito do programa de ajustamento econômico e financeiro que Portugal esteve sujeito entre 2011 e 2013, nomeadamente, a introdução de praticamente todos os produtores no regime fiscal, e a obrigatoriedade geral de contabilidade ainda que simplificada, para quem recebe apoios da União Europeia.

Este trabalho justifica-se, pois, apesar de existirem importantes estudos que analisam a eficiência no setor leiteiro em Portugal, em particular, nas Ilhas dos Açores efetuado por Silva et al. (2015); Silva e Marote (2013a); e Silva et al. (2013b); Silva et al. (2015) não existem neste momento trabalhos que analisem a eficiência nos bovinos de carne em Portugal. 
Contrariamente, verificamos que no Brasil existem importantes contributos e que a literatura é profícua em desenvolvimentos da eficiência da produção de bovinos de carne, quer com metodologias de estudo paramétricas e não paramétricas, cuja revisão é efetuada por Gomes (1999) que apresenta todos os trabalhos relevantes nesta área. Magalhães e Aragão (2006) analisaram a eficiência de produtores de leite no Estado do Ceará, Brasil, tendo concluído que há um predomínio do grupo de produtores ineficientes, representando $67,5 \%$ da amostra, sendo que é muito baixo (32,5\%) o número de produtores eficientes na amostra estudada.

Dos Santos e Quintal (2016) propõem através de técnicas de programação matemática o cálculo e as problemáticas da obtenção de uma ração com valores nutricionais adequados e de reduzido custo de produção no Brasil. Esta metodologia, uma vez ajustada, pode ser utilizada no cálculo da eficiência.

De igual forma, ultimamente, importantes contributos no cálculo da eficiência dos bovinos de corte encontram-se ainda nos Estados Unidos da América (EUA) com o trabalho de White et al. (2014) e de Qushim et al. (2016), que analisam a eficiência técnica e de escala, a produtividade marginal para inputs e outputs nos EUA através da fronteira paramétrica estocástica. Na Turquia destaca-se recentemente o estudo de Ceyhan e Kerem (2010) no caso da eficiência da produção de bovinos de carne.

Cada vez mais, a componente de sustentabilidade assume maior importância no cálculo da eficiência. Segundo Cantelle et al. (2013), a conjugação dos custos econômicos e ambientais nos setores empresarial e agrícola ainda constitui de igual forma um ponto de forte divergência. Assim, Neves et al. (2016) avaliam os impactos da produção de carne bovina na região do Pantanal no Brasil; enquanto Pereira et al. (2012) salientam que as várias atividades rurais e urbanas prejudicam o solo e, para cada uso, devem ser levadas em consideração suas propriedades e tipos de formação.

Para além de não existirem em Portugal trabalhos recentes e atualizados no âmbito da eficiência da produção de carne de bovinos, este trabalho justifica-se, pois é imperioso que a economia portuguesa possa crescer de forma sustentada. Esse crescimento poderá ser efetuado mediante o aumento da competitividade setorial em Portugal. O aumento da taxa de auto-abastecimento, pela via da produção e 
também o aumento das exportações de carne de bovino é, pois, imperioso neste âmbito. Assim, o principal objetivo deste estudo é medir os níveis de eficiência técnica de uma amostra de explorações de produção de carne de bovino, nas regiões do Alentejo e Beira Interior, em Portugal, e relacionar esses níveis de eficiência às características agrícolas e de gestão, de forma a identificar os fatores que condicionam a eficiência e, desta forma, contribuir para um aumento da eficiência deste setor no futuro.

\section{MATERIAL E MÉTODOS}

A medição da eficiência em vários setores da economia em geral, e na produção agrícola em particular, tem sido efetuada através de duas metodologias principais, nomeadamente, métodos paramétricos e não paramétricos. O primeiro efetua-se através da definição de uma fronteira de produção e o segundo através da Análise Envoltória de Dados (DEA).

Segundo Gomes (1999), em um sistema de produção identificam-se dois tipos de eficiência: técnica e econômica. Considera-se tecnicamente eficiente quando não há outro sistema que possibilite o mesmo nível de produção ao utilizar quantidade inferior ou igual de insumos. A máxima eficiência econômica ocorre quando não há outro sistema de produção alternativo que produza a mesma quantidade, a menor custo ou com maior lucro (ABREU et al., 2006). Entretanto, para que um sistema seja economicamente eficiente, é necessário que haja eficiência técnica (Gomes, 1999).

Charnes et al. (1978), e posteriormente Charnes et al. (1981), desenvolveram a metodologia DEA a partir dos trabalhos iniciais de Farell (1957). Posteriormente, a mesma foi reformulada por Banker et al. (1984), com o objetivo de possibilitar a análise no caso de retornos variáveis à escala (ABREU et al., 2006; FARE et al., 2013).

Este método não paramétrico tem sido largamente utilizado para estimar a eficiência nas unidades organizacionais em várias áreas. "DEA é uma técnica baseada em programação linear que visa à mensuração do desempenho relativo de unidades organizacionais em que ocorre presença de múltiplos insumos e produtos 
relativamente homogêneos" (ABREU et al., 2006). Neste estudo utilizamos a DEA na primeira fase para estimar a eficiência técnica. A principal razão da sua utilização é que esta não requer a especificação de uma forma funcional para a fronteira de produção e de distribuição de erros, e, portanto, evita erros de má especificação. Em segundo lugar, a DEA permite a avaliação da eficiência relativa de unidades homogêneas que possuem autonomia na tomada de decisão (Decision Making Units - DMU), o que permite a comparação de uma técnica de produção com as outras em termos de um índice de desempenho (ABREU et al., 2006). Desta forma, a DEA possibilita calcular a diferença de eficiência de cada produtor para as melhores práticas produtivas, o que pode ser avaliado a partir de observações reais de inputs e outputs de empresas eficientes (SILVA et al., 2013a).

Neste estudo, utiliza-se um modelo da DEA com orientação para outputs, porque se assume que, na ausência de quotas de produção, os gestores das explorações de bovinos têm um maior controle sobre os outputs do que sobre inputs. Utiliza-se a DEA através do Programa (DEAP) desenvolvido por Coelli (1996).

No modelo DEA orientado para outputs o valor da eficiência $\hat{\ddot{a}}_{i}$ é calculado para a exploração $i$-th, resolvendo o seguinte programa, de acordo com Latruffe et al. (2005):

$$
\begin{aligned}
& \max _{\ddot{e ̣ ~}} \hat{\ddot{a}}_{i} \\
& \text { sujeito a: } \\
& -\ddot{a}_{i} y_{i}+Y \ddot{e} \geq 0 \\
& x_{i}-X \ddot{e} \geq 0 \\
& \ddot{e} \geq 0
\end{aligned}
$$

O modelo acima é calculado a retornos constantes à escala (CRS). Para podermos obter a especificação com retornos variáveis à escala (VRS) adicionamos a restrição $\overline{\mathbf{1}} \ddot{\mathbf{e}}=\mathbf{0}$ em que $\overline{\mathbf{1}}$ é um vetor de unidade segundo Latruffe et al. (2005). A eficiência técnica calculada através de CRS é a eficiência técnica total e pode ser dividido em: a eficiência técnica pura, que representa as práticas de gestão e que é dada pela especificação prevista para VRS, e o residual que é a eficiência de escala.

Os dados obtidos neste estudo reportam-se ao ano de 2013, a 79 explorações 
agrícolas e foram obtidos através dos dados do Instituto Nacional de Estatística ${ }^{* * *}$ (INE, 2014), por serem os últimos que estavam disponíveis. As variáveis foram selecionadas com base em metodologias de autores anteriores (Abreu et al., 2006; Latruffe et al., 2005; Silva et al., 2013c). As explorações agrícolas selecionadas tinham um valor da produção de bovinos de carne (em euros) mais do que $65 \%$ do produto total da exploração que foi utilizado como a medida de output. Esta variável agregada foi construída através do número total de animais vendidos. No que diz respeito aos inputs incluímos: a superfície agrícola utilizada (SAU), em hectares (ha) como o fator terra; unidades de trabalho anuais (UTA) como o fator trabalho; amortização acrescida de juros como um fator capital; e o valor do consumo intermédio como um fator variável.

Em uma segunda fase, a estimação de um modelo de regressão linear múltipla (MLRM) é utilizado com a finalidade de determinar quais os fatores que estão a condicionar a gestão e que afetam negativamente a eficiência, de forma a que esta possa melhorar. Assim, os valores de eficiência obtidos na primeira fase são regredidos (variável dependente) em um conjunto de variáveis explicativas ou independentes. No presente estudo, infelizmente, não dispomos de variáveis que avaliem as características educacionais e/ou sociais dos agricultores (exemplo: idade, sexo, educação, número de anos de atividade agrícola, etc.), pois o conjunto de dados do INE não tem disponível este tipo de dados. Por isso, com base em trabalhos anteriores sobre a eficiência da exploração nos países do Mediterrâneo, são consideradas as seguintes variáveis explicativas: a superfície agrícola útil (SAU) das explorações de bovinos de carne é utilizada como uma variável de dimensão. $\mathrm{O}$ impacto do tamanho de eficiência técnica é um problema recorrente na literatura sobre eficiência. Por exemplo, Santos (2016) encontrou uma correlação positiva entre a dimensão e a eficiência nas explorações em Portugal.

O rácio de capital trabalho e o rácio terra trabalho são utilizadas como variáveis proxies de tecnologia. Esta evidência foi encontrada por Latruffe et al. (2005) em explorações pecuárias. O grau de integração no mercado de fatores é representado pela percentagem (\%) de mão de obra contratada no total de trabalho na exploração e pela percentagem de terras arrendadas no total da SAU, segundo

\footnotetext{
*** O Instituto Nacional de Estatística é o organismo governamental de Portugal responsável por produzir e divulgar informação estatística oficial.
} 
Dos Santos (2013). O indicador do stress financeiro na exploração agrícola é dado pelas amortizações de empréstimos mais juros mais rendas no total da produção de acordo com Latruffe et al. (2005).

Tabela 1. Dados utilizados

\begin{tabular}{lcccc}
\hline \multicolumn{1}{c}{ Variáveis } & Média & Desvio padrão & Mínima & Máximo \\
\hline Modelo DEA & & & & \\
\hline Total output (produção total) & 43,625 & 30,165 & 6,938 & 127,991 \\
Terra (ha) & 141,84 & 116,51 & 13,32 & 669,7 \\
Trabalho (UTA) & 1,335 & 0,669 & 0,41 & 4,29 \\
Capital (000 euros) & 6,062 & 4,160 & 1,346 & 19,196 \\
Consumo intermédio (000 euros) & 8,677 & 11,495 & 0,106 & 57,790 \\
Segunda fase da regressão & & & & \\
Número de animais & 43 & 36 & 5 & 263 \\
Capital/ Trabalho (euros /UTA) & 3766,21 & 4593,1 & 0 & 28453 \\
Terra / Trabalho (ha /UTA) & 106,60 & 66,78 & 9,921 & 338,55 \\
\% Trabalho contratado & 0,18 & 0,52 & 0 & 2,74 \\
\% de terra arrendada & 0,432 & 0,442 & 0 & 1 \\
Rácio de stress financeiro & 0.033 & 0.031 & 0 & 0.198 \\
\hline
\end{tabular}

Fonte: INE, 2014 e cálculos próprios.

\section{RESULTADO E DISCUSSÃO}

Os resultados da eficiência técnica, pura técnica e eficiência de escala são apresentados na Tabela 2. A percentagem de explorações agrícolas eficientes representa a percentagem de explorações com um valor de eficiência igual a uma unidade. Os resultados confirmam que apenas 8 explorações de bovinos de carne são eficientes, representando $10,2 \%$ do número total de explorações. O valor médio de eficiência técnica das explorações é de 0,72 . É possível produzir a mesma quantidade de output se reduzirmos aproximadamente $27 \%$ dos inputs. 
A eficiência técnica (CRS) é dividida em dois componentes: retornos variáveis à escala (VRS) e eficiência de escala (SCAL). A eficiência técnica do modelo a partir de retornos variáveis à escala aumenta para 0,79 e a eficiência de escala passa para 0,85 . A ineficiência de escala $(14,5 \%)$ pode ocorrer devido ao fato de $80 \%$ das explorações se encontrarem a operar em rendimentos crescentes de escala (IRS) e apenas 20\% estarem a operar em retornos decrescentes à escala (DRS).

Tabela 2. Resultados da eficiência das explorações

\begin{tabular}{lccc}
\hline & CRS & VRS & SCAL \\
\hline Media & 0,72 & 0,79 & 0,85 \\
Desvio padrão & 0,162 & 0,171 & 0,122 \\
Máximo & 1 & 1 & 1 \\
Mínimo & 0,38 & 0,45 & 0,56 \\
Explorações eficientes & 11 & 31 & 11 \\
IRS & & & $80 \%$ \\
DRS & & & $20 \%$ \\
\hline
\end{tabular}

Fonte: cálculos próprios, 2016.

Os resultados da segunda fase da estimativa da regressão múltipla são apresentados na Tabela 3. Tal como mencionado, a variável dependente representa o score de eficiência. As variáveis independentes inicialmente apresentavam-se com diferentes tipos de unidades, ou seja, ha, euros, etc. Para superar esta fonte de variação, efetuou-se a sua transformação em variáveis logaritmizadas (log). O mesmo sucedeu com a variável dependente, tratando-se, pois, de um modelo log-log.

Os resultados comprovam que a maioria das variáveis têm um impacto significativo sobre eficiência técnica da produção de bovinos. O número de efetivos pecuários tem impacto direto positivo sobre a eficiência destas explorações. $\mathrm{O}$ oposto ocorre em relação ao rácio terra/trabalho. As explorações devem reduzir o trabalho contratado e pouco habilitado a fim de obterem ganhos na eficiência, ou contratar pessoal qualificado.

A percentagem de terras arrendadas não apresenta impacto na eficiência destas explorações, o que se justifica, dado que a maioria da terra utilizada pelos produtores é própria, sendo restritos os casos de terras arrendadas nesta atividade, 
que é, por natureza, extensiva. O stress financeiro inerente ao reembolso de amortizações de empréstimos e juros tem um contributo negativo na gestão destas explorações, estando associado a explorações com elevado endividamento.

Tabela 3. Determinantes da eficiência técnica

\begin{tabular}{|l|c|}
\hline & Explorações de bovinos de carne \\
\hline Constante & $0.82 * *$ \\
\hline Variável de dimensão & $(0.25)$ \\
\hline & $2.26 \mathrm{E}-2 * *$ \\
\hline Rácio capital/trabalho & $(0.42 \mathrm{E}-2)$ \\
\hline & $2.32 \mathrm{E}-2 * *$ \\
\hline Rácio terra/trabalho & $(0.89 \mathrm{E}-2)$ \\
\hline & $-8.12 \mathrm{E}-2$ \\
\hline \% trabalho contratado & $(4.53 \mathrm{E}-2)$ \\
\hline & $-1.36 \mathrm{E}-2$ \\
\hline \% de terra arrendada & $(2.38 \mathrm{E}-2)$ \\
\hline & $-\cdots--\cdots--$ \\
\hline Rácio de stress financeiro & \\
\hline & $-41.52 * *$ \\
\hline
\end{tabular}

Fonte: Cálculos próprios, 2016. ** : 5\% significado. E-2: 10 -2. Desvio padrão entre aspas.

\section{CONSIDERAÇÕES FINAIS}

$\mathrm{O}$ artigo apresenta uma estimativa da eficiência em duas fases e permitiu determinar as principais variáveis que condicionam a eficiência de gestão das explorações portuguesas de bovinos de carne, nas principais regiões produtoras do país. As seguintes conclusões principais podem ser tiradas a partir do mesmo.

Em primeiro lugar, constatamos que a eficiência das explorações de bovinos de carne pode ser melhorada. Para tal, sugere-se que as mesmas possam atingir 
economias de escala, o que poderá ser conseguido aumentando o número de animais por hectare. Paralelamente, recomenda-se que sejam efetuados investimentos racionais em capital fixo, de forma a melhorar a tecnologia de produção. Sugerese mesmo no âmbito das políticas agrícolas portuguesas a adoção de políticas que favoreçam estas ações, nomeadamente através de políticas agrícolas da União Europeia, pois estamos face a uma atividade agro-silvo-pastoril, autóctone, milenar, típica do Mediterrâneo que deve ser preservada, pelas externalidades positivas que tem no mundo rural e na sua conservação, nomeadamente, no agro-turismo e valorização da paisagem e da sustentabilidade, bem como nas atividades a ele associadas. Paralelamente, os resultados comprovaram que esta atividade está associada a uma reduzida intensidade de mão de obra contratada e um elevado stress financeiro, o que significa que os empresários agrícolas não têm a experiência de gestão financeira necessária para tomarem decisões de investimento racional. Isto sugere que os agricultores ainda não têm a experiência de gestão necessária para decisões de investimento e de trabalho racionais.

Torna-se, pois, crucial que a gestão de explorações agrícolas siga as melhores práticas de gestão e que deixe de ser efetuada de forma empírica. Recomenda-se que o país invista em formação de excelência ao nível superior em economia e gestão agrárias. O rejuvenescimento da gestão do setor é recomendado, mediante o estímulo de políticas agrícolas e setoriais, que promovam e facilitem a integração de jovens altamente qualificados nesta atividade.

\section{REFERÊNCIAS}

ABREU, U. G. P. de; LOPES, P. S.; TORRES, R. de A.; SANTOS, H. N. Avaliação da introdução de tecnologias no sistema de produção de gado de corte no Pantanal: análise de eficiência. Revista Brasileira de Zootecnia, v. 45, n. 7, p. 409-416, set./ out. 2006.

BANKER, R.; CHARNES, A.; COOPER, W. Some models for estimating technical and scale inefficiencies in data envelopment analysis. Management Science, v. 30, p. 1078-1092, 1984. 
CHARNES, A.; COOPER, W.; RHODES, E. Measuring the Efficiency of Decision Making Units. European Journal of Operational Research v. 2, p. 429-444. 1978.

CHARNES, A.; COOPER, W.; RHODES, E. Evaluating Program and Managerial Efficiency: An Application of Data Envelopment Analysis to Program Follow Through. Management Science, v. 27, n. 6, p. 668-697, 1981.

CANTELLE, T. D.; LIMA, L. M. E. S.; REIS, R. P.; MAGALHÃES, L. C. A. Competitividade na Agricultura Comercial Versus Sustentabilidade Ambiental. Revista em Agronegócio e Meio Ambiente, v. 6, n. 1, p. 171-188, jan./abr. 2013.

COELLI, T. A guide to DEAP version 2.1: a data envelopment analysis program. Armidale: University. 49 p. Jan. 1996. Disponível em: http://www.owlnet.rice.edu/ econ380/DEAP.PDF. Acesso em: 23 jan. 2016.

CEYHAN, V.; KEREM, H. Economic efficiency of cattle-fattening farms in Amasya province, Turkey. Journal of Animal and Veterinary Advances, v. 9, n. 1, p. 6069, 2010.

DOS SANTOS, M.; QUINTAL, R. S. Problema de Programação Linear da Dieta Aplicado à Nutrição de Suínos. Revista em Agronegócio e Meio Ambiente, Maringá, v. 9, n. 2, p. 251-271, abr./jun. 2016.

DOS-SANTOS, M. J. P. L. Segmenting Farms in European Union. Agricultural Economics, v. 59, p. 49-57, 2013.

DOS-SANTOS, M. J. P. L.; HENRIQUES, P. D. S.; FRAGOSO, R. M. S.; CARVALHO, M. L. S. A competitividade do regadio de Alqueva: O caso do Bloco de rega do Monte Novo, Revista de Economia e Sociologia Rural, v. 50, n. 1, p. 107-118, 2012.

DOS-SANTOS, M. J. P. L.. Smart cities and urban areas - Aquaponics as innovative urban agriculture. Urban Forestry \& Urban Greening, v. 20, 402-406, 2016.

FARREL, M. J. The Measurement of Productive Efficiency. Journal of the Royal Statistical Society, v. 120, p. 253-281, 1957. 
FARE, R.; GROSSKOPF, S.; LOVELL, C. A. The Measurement of Efficiency of Production. In: Springer Science: Business Media, Kluwer-Nijhoff publishing, Boston, 2013. p. 78-121.

FONSECA, A. M.; DIZ, H. M.; DOS-SANTOS, M. J. P. L.. El crowdfunding como financiación del periodismo de investigación en Portugal. Palabra Clave, v. 19, n. 3 , p. 893-918, 2016.

GOMES, A. P. Impactos das transformações da produção de leite no número de produtores e requerimentos de mão de obra e capital. 1999. $161 \mathrm{f}$. (Doutorado em Economia Rural) - Universidade Federal de Viçosa, Viçosa, 1999.

INE - INSTITUTO NACIONAL DE ESTATÍSTICA. Estatísticas agrícolas, 2014.

LATRUFFE, L.; BALCOMBE, K.; DAVIDOVA, S.; ZAWALINSKA, K. Technical and Scale Efficiency of Crop and Livestock Farms in Poland: Does Specialisation Matter? Agricultural Economics, v. 32, n. 3, p. 281-296, 2005.

MAGALHAES, K. A.; CAMPOS, R. T.. Eficiência técnica e desempenho econômico de produtores de leite no Estado do Ceará, Brasil. Revista Economia e Sociologia Rural, Brasília, v. 44, n. 4, p. 695-711, Dec. 2006.

NEVES, D. A. L.; SOARES, J. P. G.; CARVALHO, J. M.. Produção de Carne Bovina Orgânica: Uma Avaliação dos Impactos Socioeconômicos na Região do Pantanal do Brasil. Revista em Agronegócio e Meio Ambiente, v. 9, n. 1, p. 71-92, jan./mar. 2016.

PEREIRA, M. T.; GIMENES, M. L.; DA SILVA, F. F. F.; ZANATTA, O. A. Desenvolvimento de indicador de qualidade de áreas verdes urbanas (IQAVU) e aplicação em cidades paranaenses. Revista em Agronegócio e Meio Ambiente, v. 5, n. 1, p. 132-159, jan./abr. 2012.

QUSHIM, B.; GILLESPIE, J.; PAUDEL, K.; MCMILLIN, K. Technical and scale efficiencies of meat goat farms in the USA. Applied Economics, v. 48, n. 7, p. 608-620, 2016. 
SILVA, E.; SANTOS, C.; MENDES, A. B. Animal grazing system efficiency. Efficiency Measures. In: Efficiency Measures in the Agricultural Sector. Springer Netherlands, 2013b. 83-91.

SILVA, E.; ARZUBI, A.; BERBEL, J. An application of data envelopment analysis (DEA) in Azores dairy farms. Efficiency Measures in the Agricultural Sector. Springer, Dordrecht, 2013c. 73-81.

SILVA, E.; MARTA-COSTA, A. A.; BERBEL, J. The Objectives and Priorities for the Azorean Dairy Farmers' Decisions. In: The Agricultural Economics of the 21st Century. Springer International Publishing, 2015. 137-156.

WHITE, R. R.; BRADY, M.; CAPPER, J. L.; JOHNSON, K. A. Optimizing diet and pasture management to improve sustainability of US beef production. Agricultural Systems, v. 130, p. 1-12, 2014.

Recebido em: 24/08/2016

Aceito em: 28/08/2018 